\title{
A numerical study of the Martian atmospheric convection with a two-dimensional anelastic model
}

\author{
Masatsugu Odaka ${ }^{1}$, Kensuke Nakajima ${ }^{2}$, Shin-ichi Takehiro ${ }^{2}$, Masaki Ishiwatari ${ }^{3}$, and Yoshi-Yuki Hayashi ${ }^{1}$ \\ ${ }^{1}$ Graduate School of Mathematical Sciences, University of Tokyo, Japan \\ ${ }^{2}$ Department of Earth and Planetary Sciences, Faculty of Science, Kyushu University, Japan \\ ${ }^{3}$ Graduate School of Environmental Earth Science, Hokkaido University, Japan
}

(Received August 2, 1997; Revised February 20, 1998; Accepted February 26, 1998)

Thermal convection of the Martian lower atmosphere is examined by the use of a two-dimensional anelastic model with a resolution fine enough to describe convection eddies.

For a homogeneous radiative cooling of $50 \mathrm{~K} /$ day given in the layer below $5 \mathrm{~km}$, a layer of time-dependent convection develops up to about $6 \mathrm{~km}$ in height. The intensity of realized vertical winds ranges up to $20 \mathrm{~m} / \mathrm{s}$. The dust, which is injected into the lowest layer and treated as a passive tracer, is transported immediately in the convection layer and mixed uniformly.

The intensity of the horizontal winds near the surface reaches about $10 \mathrm{~m} / \mathrm{s}$, which, combined with large-scale motions, is expected to contribute to the dust injection into the atmosphere.

\section{Introduction}

In the Martian atmosphere, dust is regarded as an important factor in determining the thermal and circulation structures (Zurek et al., 1992). However, the processes of injection and mixing up of dust into the atmosphere have not been intensely investigated. The major process of the vertical mixing in the lower Martian atmosphere is the thermal convection which is driven by radiation and heat flux from the surface. The existence of thermal convection is exemplified by Hess et al. (1977) where a variation of winds with a frequency of about 20 minutes and the amplitude of about $5 \mathrm{~m} / \mathrm{s}$ is shown from the wind data of Viking landers I and II. In the researches into the Martian atmosphere, one-dimensional radiative convective models and general circulation models (GCM's) have been utilized. In these models, the thermal convection cannot be explicitly treated; the effects of thermal convection are parameterized as vertical diffusion processes and/or simplified adjustment processes (convective adjustment).

The GCM's with parameterized thermal convection cannot produce sufficient wind velocity near the surface for dust injection, as is pointed out by Wilson and Hamilton (1996) in the investigation of global dust storms. When the atmosphere is already under a dusty condition, the instantaneous wind velocity in GCM can exceed the threshold value as is exemplified by Greeley et al. (1993) for $\tau=5.0$. However, starting from a clear-sky condition $(\tau \leq 1)$, the GCM's do not produce a sufficient amount of surface wind velocity to boot a global dust storm.

In order to evaluate the wind velocity near the surface, it is necessary to investigate the detailed circulation structure

Copy right (C) The Society of Geomagnetism and Earth, Planetary and Space Sciences (SGEPSS); The Seismological Society of Japan; The Volcanological Society of Japan; The Geodetic Society of Japan; The Japanese Society for Planetary Sciences. of the thermal convection. There have been no research activities into explicitly calculating the thermal convection of the Martian atmosphere. In the terrestrial atmosphere, the corresponding structure driven by radiative forcing is the cumulus convection. In investigations into revealing the possible circulation structures and moisture distributions in the cumulus convection, two-dimensional numerical models have been utilized (e.g., Nakajima and Matsuno, 1988; Nakajima, 1994; Tao et al., 1996). In two-dimensional models, the larger spatial extent and the higher spatial resolution can be easily obtained by restricting the spatial dimension. Because of those merits, two-dimensional models have been often chosen instead of three-dimensional models. We are anticipating that the same method is also useful in the investigation of the Martian atmosphere. The Martian atmosphere can be treated as a dry atmosphere, since the latent heat release does not play a significant role in the circulation systems. In order to understand the structures of dry convection, we have to follow the stream of studies on cumulus convection, since the structures of radiative convection in a dry atmosphere have been rarely examined in meteorology so far.

As an attempt to understand the dry convection driven by radiative forcing, we will perform numerical calculations using a two-dimensional model. We will examine the cell structure and circulation intensity of the realized dry convection. The resultant convective mixing of dust will be demonstrated.

In this study, the thermal convection is driven by fixed cooling the surface fluxes given by a constant bulk coefficient on a ground surface with a fixed temperature. This simple setup of convection is far from the reality of the Martian atmosphere. However, our setup contains minimal components of dry convection and will present some of the essential features of the Martian atmospheric convection. At 
the same time, the present study is the necessary first step with which the results of more sophisticated future studies will be compared.

\section{Model}

We utilize the two-dimensional anelastic system of Ogura and Phillips (1962), i.e., a Boussinesq system that permits the vertical variation of the basic state. This is the system that has been often utilized in various models for investigating terrestrial deep convection (e.g., Fovell and Ogura, 1988; Vallis et al., 1997): The governing equations are given as

$$
\begin{aligned}
& \frac{\partial\left(\rho_{0} u\right)}{\partial x}+\frac{\partial\left(\rho_{0} w\right)}{\partial z}=0, \\
& \frac{\partial u}{\partial t}+u \frac{\partial u}{\partial x}+w \frac{\partial u}{\partial z} \\
& =-c_{p} \theta_{0} \frac{\partial \pi}{\partial x}+\frac{\partial}{\partial x}\left(K \frac{\partial u}{\partial x}\right)+\frac{1}{\rho_{0}} \frac{\partial}{\partial z}\left(\rho_{0} K \frac{\partial u}{\partial z}\right),
\end{aligned}
$$

$$
\begin{aligned}
& \frac{\partial w}{\partial t}+u \frac{\partial w}{\partial x}+w \frac{\partial w}{\partial z} \\
& =-c_{p} \theta_{0} \frac{\partial \pi}{\partial z}+g \frac{\theta}{\theta_{0}}+\frac{\partial}{\partial x}\left(K \frac{\partial w}{\partial x}\right)+\frac{1}{\rho_{0}} \frac{\partial}{\partial z}\left(\rho_{0} K \frac{\partial w}{\partial z}\right)
\end{aligned}
$$

$$
\begin{aligned}
& \frac{\partial \theta}{\partial t}+u \frac{\partial \theta}{\partial x}+w \frac{\partial\left(\theta+\theta_{0}\right)}{\partial z} \\
& =\frac{\partial}{\partial x}\left(K \frac{\partial \theta}{\partial x}\right)+\frac{1}{\rho_{0}} \frac{\partial}{\partial z}\left(\rho_{0} K \frac{\partial\left(\theta+\theta_{0}\right)}{\partial z}\right) \\
& +\frac{Q_{\mathrm{rad}}}{\left(p_{0} / p_{00}\right)^{R / c_{p}}},
\end{aligned}
$$

where $x$ and $z$ are the horizontal and vertical coordinates, $u$ and $w$ are the corresponding velocity components, $\rho$ is density, $p$ is pressure, $\theta$ is the deviation of potential temperature from the basic state, and $\pi$ is the deviation of $\left(p_{0} / p_{00}\right)^{R / c_{p}}$ from the basic state. Thermodynamic variables of the basic state depend only on $z$ and are denoted by the subscript $0 . p_{00}, c_{p}$ and $R$ are the constant reference pressure, specific heat at constant pressure, and gas constant. Their values are $7 \mathrm{hPa}, 734.9 \mathrm{~J} / \mathrm{kg} / \mathrm{K}, 189.0 \mathrm{~J} / \mathrm{kg} / \mathrm{K}$, respectively. $Q_{\text {rad }}$ is the cooling rate that crudely represents the effect of radiative processes. $K$ is the coefficient of turbulent mixing, whose value is evaluated by following Klemp and Wilhelmson (1978). The coefficient is given as $K=0.2 \sqrt{\varepsilon} l$. $l$ is the characteristic mixing length which is determined as the smaller value of the grid size or the height of the grid point. $\varepsilon$ is the subgrid turbulent kinetic energy whose prognostic equation is given as follows:

$$
\begin{aligned}
\frac{d \varepsilon}{d t}= & -g \frac{K}{\theta_{0}} \frac{\partial\left(\theta+\theta_{0}\right)}{\partial z} \\
& +K\left\{\left(\frac{\partial u}{\partial z}+\frac{\partial w}{\partial x}\right)^{2}+2\left[\left(\frac{\partial u}{\partial z}\right)^{2}+\left(\frac{\partial w}{\partial x}\right)^{2}\right]\right\} \\
& -0.2 \frac{\varepsilon^{3 / 2}}{l}+\frac{\partial}{\partial x}\left(K \frac{\partial \varepsilon}{\partial x}\right)+\frac{1}{\rho_{0}} \frac{\partial}{\partial z}\left(\rho_{0} K \frac{\partial \varepsilon}{\partial z}\right) .
\end{aligned}
$$

In this study, dust is radiatively inactive and is treated only as a passive tracer. The terminal fall velocity of dust is ignored, which is a good approximation for the short period of time integration performed in this study. According to the estimation by Conrath (1975), the terminal velocity of a dust particle with a diameter of $1 \mu \mathrm{m}$ is about $10^{-3} \mathrm{~m} / \mathrm{sec}$, by which is falls less than $100 \mathrm{~m}$ for a period of one day. Time evolution of dust mixing ratio $q$ is expressed as

$$
\frac{\partial q}{\partial t}+u \frac{\partial q}{\partial x}+w \frac{\partial q}{\partial z}=\frac{\partial}{\partial x}\left(K \frac{\partial q}{\partial x}\right)+\frac{1}{\rho_{0}} \frac{\partial}{\partial z}\left(\rho_{0} K \frac{\partial q}{\partial z}\right)
$$

Equations (1) to (6) are discretized on the staggered grid. Both the horizontal and vertical grid intervals are $100 \mathrm{~m}$. The lowest level of the horizontal wind is placed at $50 \mathrm{~m}$ height. The model domain is extended to a width of $51.2 \mathrm{~km}$ and to a height of $10.0 \mathrm{~km}$.

Radiative forcing $Q_{\text {rad }}$ is introduced as a uniform cooling rate in the lower layer of the atmosphere. The choice of negative value of $Q_{\text {rad }}$ might confuse those who are familiar with the daytime condition of the Martian atmosphere. However, under the radiative-convective equilibrium state, convection should develop so that convective heating balances with radiative cooling. According to the one-dimensional calculation by Haberle et al. (1993), the parameterized convective heating in daytime is $50 \mathrm{~K} /$ day in the region where convection occurs. In the standard experiment of our study, expecting the emergence of convection with the same intensity as that of Haberle et al. (1993), the radiative cooling is assumed to be $50 \mathrm{~K} /$ day below $5 \mathrm{~km}$ in the atmosphere. Experiments with cooling rates of $6.25 \mathrm{~K} /$ day, $12.5 \mathrm{~K} /$ day, $25 \mathrm{~K} /$ day and $100 \mathrm{~K} /$ day are also performed to investigate the dependency of the convective motions on the intensity of radiative cooling.

The horizontal boundary conditions are cyclic. At the top and bottom of the atmosphere, the vertical velocity is assumed to be 0 . The momentum flux from the surface due to the friction is calculated by the bulk formula. A free-slip condition is applied at the top of the atmosphere. The ground temperature $T_{s}$ is fixed to be $270 \mathrm{~K}$. This value is almost the same as the observed Martian surface temperature in daytime at the subsolar point (Martin et al., 1979). The heat flux from the surface is given by the bulk formula. The bulk coefficients of both momentum and heat are assumed to be $C_{d}=0.01$. Dust is injected in the lowest level of the model (50 m height) with a constant mass flux of $10^{-8} \mathrm{~kg} / \mathrm{m}^{2} / \mathrm{s}$ which 
is arbitrarily adapted from the value utilized by Murphy et al. (1993).

The initial atmospheric temperature profile is adiabatic from the surface $(245 \mathrm{~K})$ to $5 \mathrm{~km}$ height $(220 \mathrm{~K})$, and is isothermal above that. This initial temperature profile is adapted from the result of the one-dimensional calculation by Haberle et al. (1993). The basic state is calculated from this initial temperature profile by assuming hydrostatic equilibrium. The numerical integration is performed for 12 hours or longer, which is a duration one order of magnitude longer than the timescale of the development of convection cell, as will be described later.

\section{Results}

Figure 1 shows the distributions of vertical and horizontal winds, potential temperature, and dust mixing ratio after 12 hours of integration for the case with a radiative cooling of $50 \mathrm{~K} /$ day. The vertical velocity field is characterized by the appearance of several updrafts and downdrafts placed alternately. Their vertical extent is about $6 \mathrm{~km}$, which is nearly the same as the typical distances between the neighboring pairs of updrafts and downdrafts in the model; the aspect ratio of the convection cells is roughly one. The intensities of the vertical and horizontal winds reach about $20 \mathrm{~m} / \mathrm{s}$ and about $10 \mathrm{~m} / \mathrm{s}$, respectively. In spite of the existence of
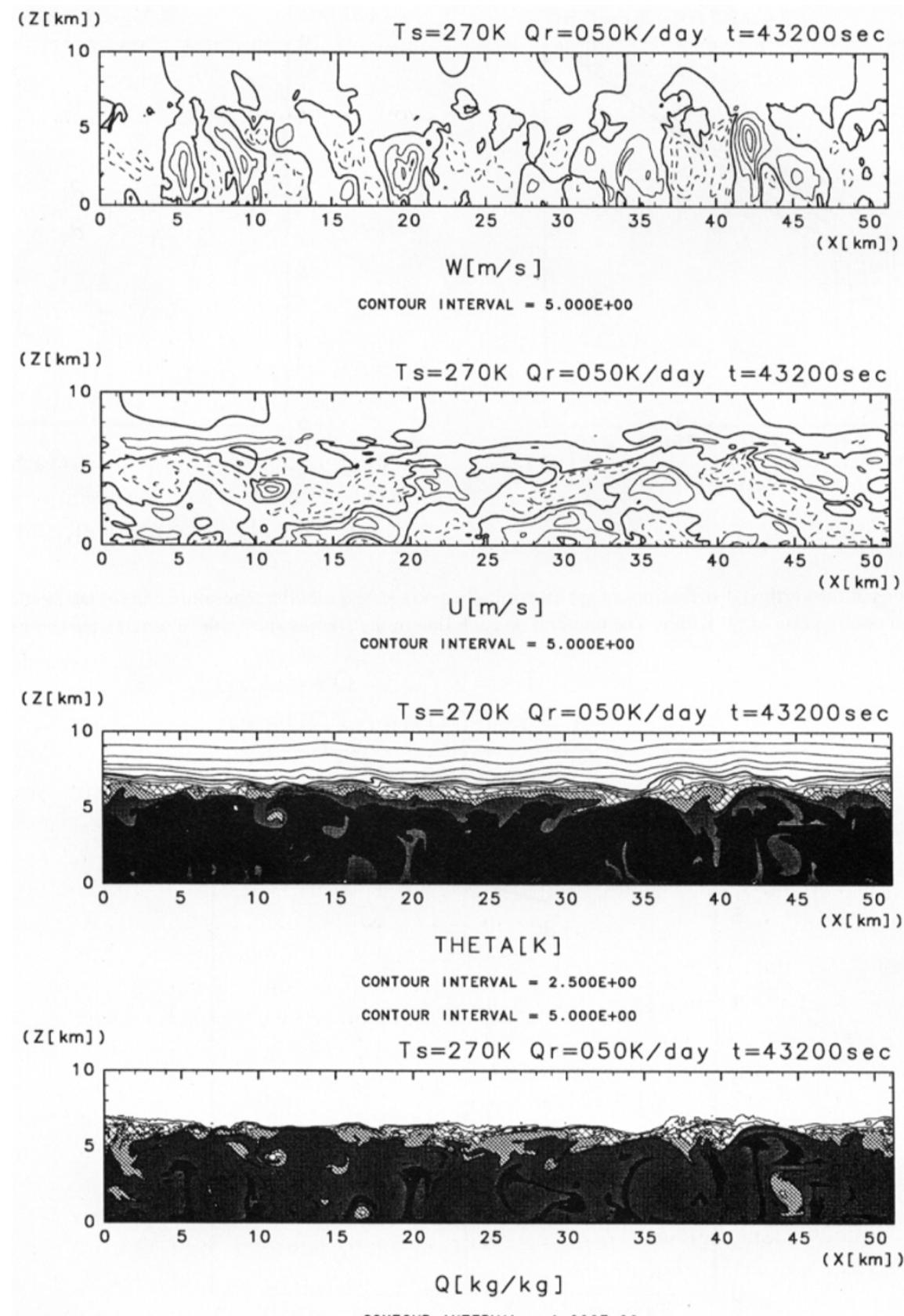

Fig. 1. Appearance of convection after 12 hours of integration for the case with a cooling rate of $50 \mathrm{~K} /$ day. Each figure represents vertical wind, horizontal wind, potential temperature, and dust mixing ratio from the top to the bottom, respectively. The contour interval of the velocities is 5 $\mathrm{m} / \mathrm{s}$. The contour interval of potential temperature is $5 \mathrm{~K}$ above $250 \mathrm{~K}$. Below $245 \mathrm{~K}$, the areas between the contours of $2.5 \mathrm{~K}$ interval are hatched with different patterns. The contour interval of the dust mixing ratio is $1.0 \times 10^{-6} \mathrm{~kg} / \mathrm{kg}$. The areas between the contours above $2.0 \times 10^{-6} \mathrm{~kg} / \mathrm{kg}$ are hatched with different patterns. 
surface friction, the horizontal wind near the ground surface is as strong as those aloft. The potential temperature is well mixed in the convection layer. However, close examination reveals the existence of warm mushroom-shaped plumes ascending from the surface layer where the potential temperature is higher than that of the convection layer. The convection is not steady. The characteristic timescale of the fluctuation is about 1 hour, which is the lifetime of the rising plumes; that is not the vertical travel time of fluid particles (i.e., $6 \mathrm{~km} /(15 \mathrm{~m} / \mathrm{s})=400 \mathrm{sec})$. The ascending plumes carry the dust from the surface layer where the mixing ratio of dust is high. The dust, after being imported into the convection layer, is mixed rapidly by the irregular convective motion both horizontally and vertically.

Figure 2(a) shows the time evolution of the horizontally averaged potential temperature. Only after 2 hours of integration, is the potential temperature vertically well mixed below the height of $5 \mathrm{~km}$; this rapid evolution results from the neutral layer given initially. A shallow unstable layer below the height of $300 \mathrm{~m}$ is notable. It is the layer of subgrid scale heat conduction from the surface to the convection layer. As time goes on, the convection layer is slowly cooled because of the imbalance between the amount of radiative cooling and that of the heat supply from the surface. At the

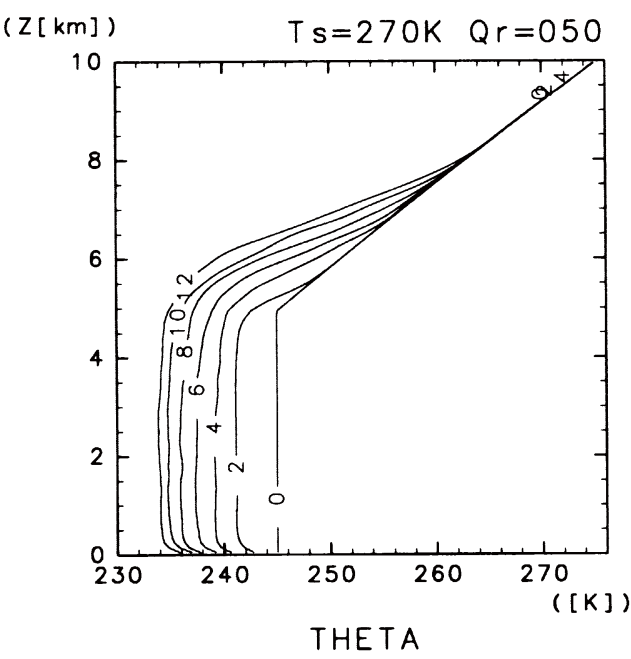

(a)

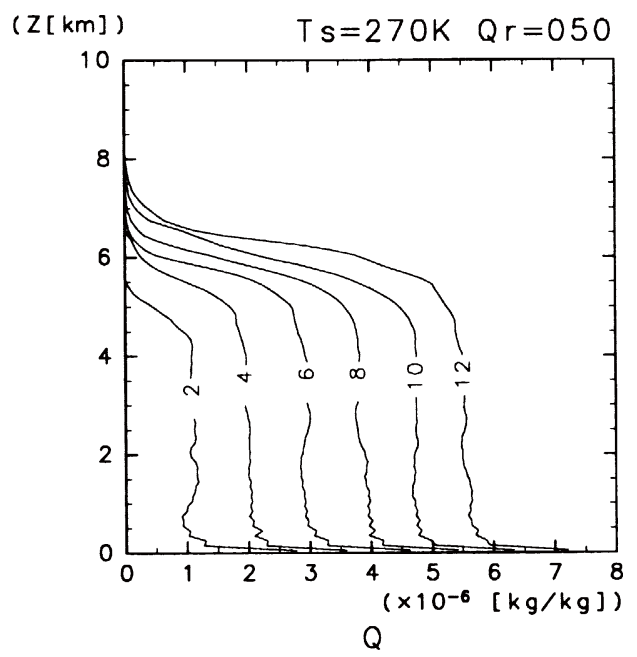

(b)

Fig. 2. Temporal evolutions of the vertical distributions of (a) horizontally averaged potential temperature and (b) horizontally averaged dust mixing ratio for the case with a cooling rate of $50 \mathrm{~K} /$ day. The numeral on each line in the figures shows the elapsed time (hours) from the initial state.

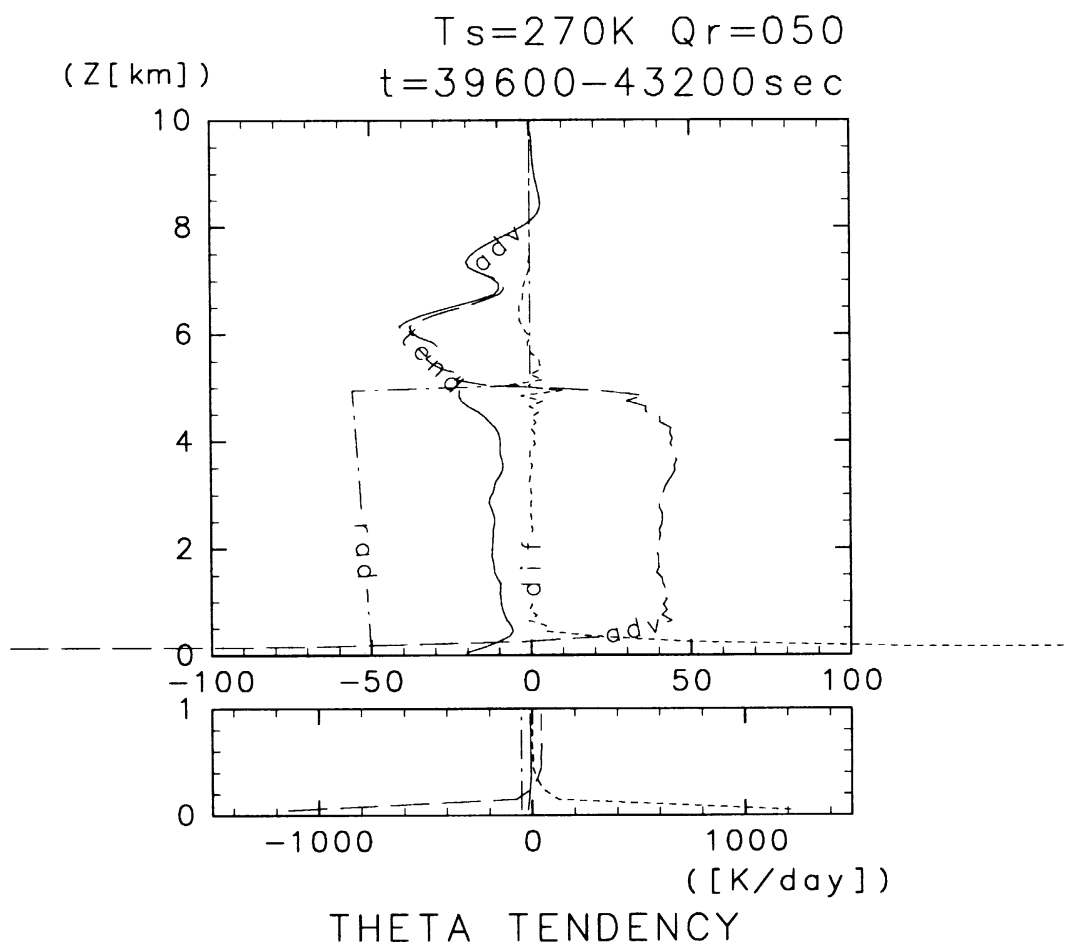

Fig. 3. Contributions of the terms of the horizontally averaged heat equation. The values are plotted after averaging over a time period between 11 and 12 hours. The lower panel shows the magnified profiles near the surface. Solid line indicates the tendency of potential temperature. Dotted, broken and broken dotted lines indicate diffusive, advective, and radiative heating terms, respectively. 
same time, the vertical extent of the convective layer increases by the collective results of the penetration of plumes.

Figure 2(b) shows the time evolution of the horizontally averaged dust mixing ratio. After 2 hours of integration, the dust supplied from the surface distributes almost uniformly below the height of $5 \mathrm{~km}$. The exception is the shallow surface layer where a larger dust mixing ratio is observed. Since the sedimentation of dust is ignored, the dust accumulates monotonically in the atmosphere. The vertical homogeneity of dust in the convection layer persists during the time integration; this is quite resonable because the timescale of the increase in the total dust amount (i.e., the length of the time integration) is longer than the timescale of dust mixing in the convective layer (i.e., the vertical travel time of the air parcel).

Figure 3 shows the contributions of the terms in the horizontally averaged heat equation. The values are plotted after averaging over a time period between 11 and 12 hours. At the lowest levels, the main contributors are strong diffusive heating and advective cooling. In the layer between the height of $500 \mathrm{~m}$ and $5 \mathrm{~km}$, the large fraction of the radiative cooling is compensated by the advective heating; the remainder is small cooling. In the layer above the height of 5 $\mathrm{km}$, the dominant contributor is the advective cooling which is caused by the penetration of ascending plumes.

Figure 4 shows the time evolution of the potential temperature during the initial two hours to demonstrate the extent to which our model can resolve the formation of the thermal plumes. Since we have initially placed the atmospheric temperature at about $30 \mathrm{~K}$ lower than the ground temperature below, a thermal boundary layer forms near the surface at the very beginning of the integration. At $t=30$ minutes, the thermal boundary layer extends up to about 250 $\mathrm{m}$ height (two to three grid levels), where Rayleigh-Taylor instability occurs to produce smallscale plumes with a size of about $500 \mathrm{~m}$. As time goes on, these plumes are organized into larger plumes. After 2 hours, the convective motion is dominated by the circulation with a scale of about $5 \mathrm{~km}$. The upward motion of the small plumes from the surface thermal boundary layer can be observed only at the low level convergence zone of the circulation cell with a scale of about $5 \mathrm{~km}$.

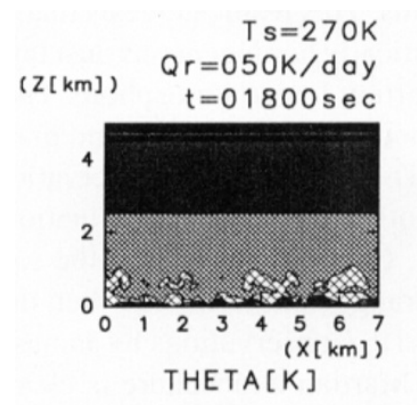

CONTOUR INTERVAL - 5.000E-02

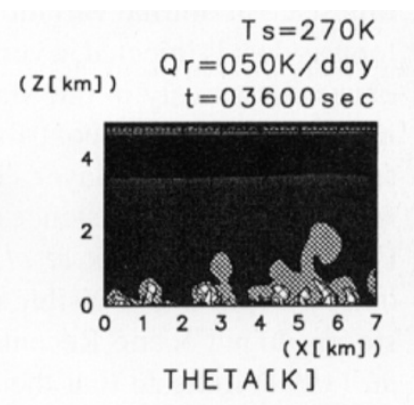

CONTOUR INTERVAL $=2.500 E-01$
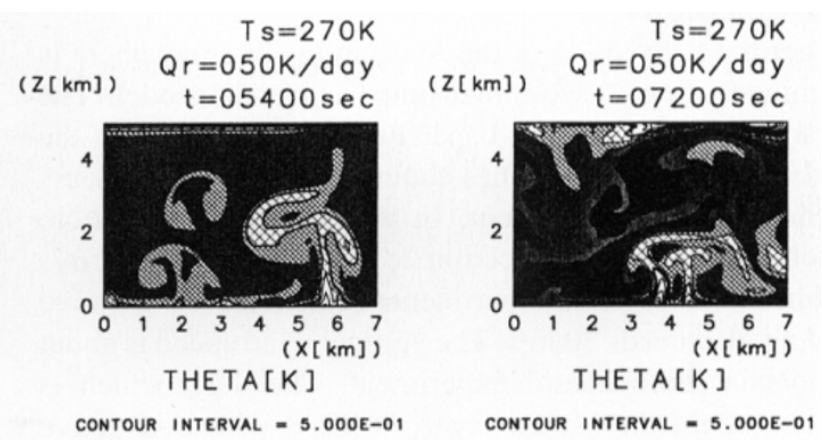

Fig. 4. Potential temperature fields at $t=30 \mathrm{~min}, t=60 \mathrm{~min}, t=90 \mathrm{~min}$ and $t=120 \mathrm{~min}$ for the case with a cooling rate of $50 \mathrm{~K} /$ day. Note that only a small part of the whole region is shown. The contour intervals are $0.05 \mathrm{~K}$ for $t=30 \mathrm{~min}, 0.25 \mathrm{~K}$ for $t=60 \mathrm{~min}$ and $0.5 \mathrm{~K}$ for $t=90 \mathrm{~min}$ and $t=$ $120 \mathrm{~min}$. Note that the same tone patterns of the different panels do not always represent the same potential temperature.

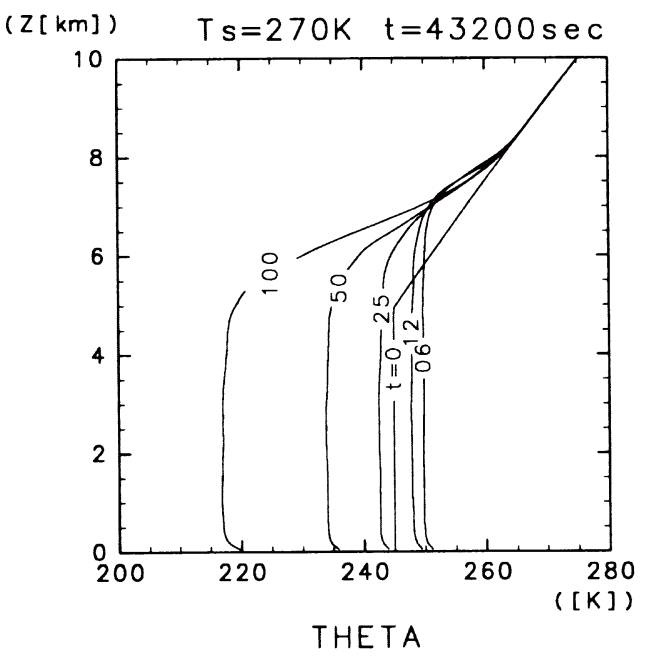

(a)

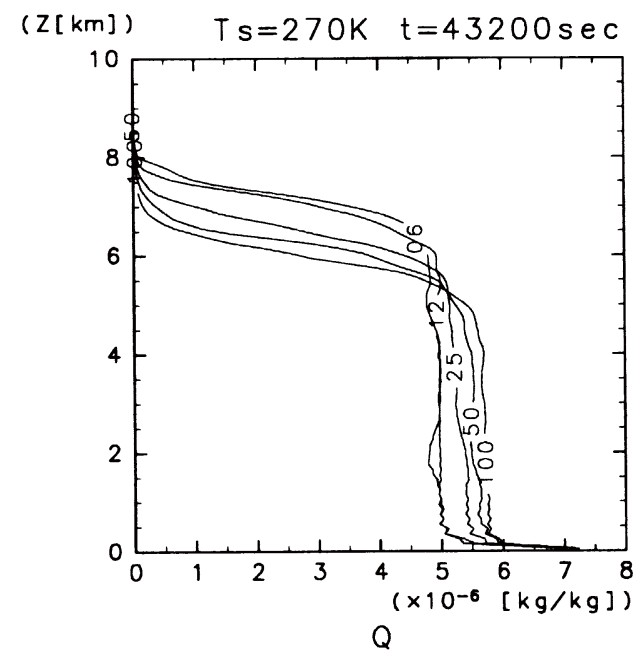

(b)

Fig. 5. The vertical distributions of (a) horizontally averaged potential temperature and (b) horizontally averaged dust mixing ratio for various cooling rates after 12 hours of integration. The numerals in the figures show the cooling rates. The initial potential temperature profile is denoted by " $t=$ 0 ". 
Figure 5(a) shows the vertical profiles of horizontally averaged potential temperature after 12 hours of integration for experiments with different intensities of radiative cooling. As the radiative cooling increases, the potential temperature of the convection layer decreases and the vertical extent of the layer becomes slightly smaller. The decrease of potential temperature is caused by the requirement that the surface heat flux should follow the increase of total radiative cooling. Since the amplitudes of wind velocity near the surface vary weakly among the experiments, a larger difference between the surface air temperature and the ground temperature is necessary to receive the larger amount of heat from the ground surface. Hence, the temperature of the convection layer must decrease in relation to the increase of the cooling rate. Note that the calculated states shown in Fig. 5(a) do not reach the thermal equilibria.

The vertical profiles of horizontally averaged dust distribution is insensitive to the radiative cooling rate. As shown in Fig. 5(b), dust is distributed almost uniformly with a mixing ratio of $6 \times 10^{-6} \mathrm{~kg} / \mathrm{kg}$ in all cases. The small difference of the mixing ratio is caused by a slight change in the depth of the convection layer.

\section{Discussions}

Thermal convection of the Martian lower atmosphere is examined using a two-dimensional anelastic model. The calculated horizontal wind speed at the lowest level of the model (50 m height) reaches about $10 \mathrm{~m} / \mathrm{s}$ within 12 hours in the standard experiment. In an extended calculation, which is not shown in Section 3, it reaches about $20 \mathrm{~m} / \mathrm{s}$ within 24 hours. In the experiments with increased radiative cooling, it exceeds $30 \mathrm{~m} / \mathrm{s}$. The vertical wind speed is about $20 \mathrm{~m} / \mathrm{s}$ in the standard experiment. The dust, which is injected from the lowest layer, is lifted with convective plumes, and is mixed homogeneously within a timescale of about one to two hours.

The validity of our model resolution is supported by the small contribution of the turbulent diffusion terms in the equations of motion. Even near the surface, the values of the turbulent diffusion coefficient are less than $30 \mathrm{~m}^{2} / \mathrm{s}$, which corresponds to the turbulent velocity scale $(\sqrt{2 \varepsilon})$ of $\sim 2 \mathrm{~m} /$ $\mathrm{s}$. This is quite smaller than the magnitude of the calculated wind velocity $(\sim 10 \mathrm{~m} / \mathrm{s})$. These values show that the unresolved nonlinear effect is small.

Near the surface, the present resolution may not be sufficient to represent the expected rapid vertical variations of the temperature profile. We have performed supplementary calculations with enhanced resolution near the surface. The grid points below the $150 \mathrm{~m}$ height level are located at 85.36 $\mathrm{m}, 60.36 \mathrm{~m}, 42.68 \mathrm{~m}, 30.18 \mathrm{~m}, 21.34 \mathrm{~m}, 15.08 \mathrm{~m}$, and 6.25 $\mathrm{m}$. The bulk coefficient $C_{d}$ is set to be 0.023 so as to reproduce the time evolution of the horizontally averaged potential temperature in the convective layer of the standard experiment. As is expected, steep vertical temperature gradients appear near the surface where horizontally averaged potential temperature ranges from $241.7 \mathrm{~K}$ at $6.25 \mathrm{~m}$ to $236.9 \mathrm{~K}$ at $150 \mathrm{~m}$ after 12 hours of time integration. Nevertheless, the size and intensity of convective motions have not been altered. The potential temperature deviations of the ascending plumes are the same as those of the standard experiment, although the potential temperatures of the newly resolved lowest layers have been increased. This suggests that the vertical resolution of the standard experiment is sufficient to represent the generation of major plumes.

The magnitude of the obtained horizontal wind speed near the surface is insufficient for dust injection into the atmosphere. This situation is the same as that of Wilson and Hamilton (1996), where the lowest level (100 m height) wind obtained by GCM is smaller than $30 \mathrm{~m} / \mathrm{s}$, which is the threshold wind value at this height necessary to raise dust from the surface (Greeley et al., 1980). However, the results of present research indicate that surface winds of this magnitude are easily realized whenever thermal convection exists. We may expect that the coexistence of thermal convection and large-scale circulation increases the absolute value of the surface wind and enables efficient dust injection. The effect of surface wind induced by thermal convection may be implemented in GCM's, for instance, by increasing the frictional velocity, which is used in the evaluation of the dust injection, by the corresponding amount diagnosed by the turbulent closure formula.

The calculated timescale of the vertical mixing of dust is about one to two hours, which is somewhat smaller than the timescale of diurnal variations. This result suggests that, so long as dust is injected, a vertically homogeneous dust layer exists extensively in the Martian lower atmosphere. Thermal convection may contribute to the formation and maintenance of the dust layer. There are several observations which suggest the existence of dust under normal situations. For example, Pollack et al. (1979) showed that the total optical depth in the visible range is at least 0.3 when dust storms do not occur. Recent HST observations by James et al. (1994) indicate that the Martian atmosphere is clearer than the 1970's but still the optical depth ranges from 0.1 to 0.2 . At the present stage, we cannot identify the origin of the values of those observed optical depths with the thermal convection of the lower atmosphere.

It is necessary to perform convection calculations using a more realistic model, where explicit calculations of the surface thermal budget and the radiative processes, including the property of dust, are implemented. The interesting issues to be investigated are the effect of the diurnal variation of radiative forcing and the interaction between the existence of dust and the convective motions. The results in this paper might be altered when the effects mentioned above are considered. These are the problems for further investigation.

Acknowledgments. The authors wish to thank the anonymous referees for their useful comments. The numerical codes and software environments were constructed using the resources of GFD DENNOU Club. The calculations were performed by FACOM VPP 500 at the Center for PLAnning and INformation Systems, Institute of Space and Astronautical Science.

\section{References}

Conrath, B. J., Thermal structure of the Martian atmosphere during the dissipation of the dust storm of 1971, Icarus, 24, 36-46, 1975.

Fovell, R. G. and Y. Ogura, Numerical simulation of a midlatitude squall line in two dimensions, J. Atmos. Sci., 45, 3846-3879, 1988.

Greeley, R., R. Leach, B. R. White, J. D. Iversen, and J. B. Pollack, 
Threshold wind speeds for sand on Mars: wind tunnel simulations, Geophys. Res. Lett., 7, 121-124, 1980.

Greeley, R., A. Skypeck, and J. B. Pollack, Martian aeolian features and deposits: comparisons with general circulation model results, $J$. Geophys. Res., 98, 3183-3196, 1993.

Haberle, R. M., H. C. Houben, R. Hertenstein, and T. Herdtle, A boundarylayer mode for Mars: comparison with Viking lander and entry data, $J$ Atmos. Sci., 50, 1544-1559, 1993.

Hess, S. L., R. M. Henry, C. B. Leovy, J. A. Ryan, and J. E. Tillman, Meteorological results from the surface of Mars: Viking I and II, $J$ Geophys. Res., 82, 4559-4574, 1977.

James, P. B., R. T. Clancy, S. W. Lee, L. J. Martin, R. B. Singer, E. Smith, R. A. Kahn, and R. W. Zurek, Monitoring Mars with the Hubble space telescope: 1990-1991 observations, Icarus, 109, 79-101, 1994.

Klemp, J. B. and R. B. Wilhelmson, The simulation of three-dimensional convective storm dynamics, J. Atmos. Sci., 35, 1070-1096, 1978.

Martin, T. Z., A. R. Peterfreund, E. D. Miner, H. H. Kieffer, and G. E. Hunt, Thermal infrared properties of the Martian atmosphere 1. Global behavior at 7, 9, 11 and 20- $\mu \mathrm{m}, J$. Geophys. Res., 84, 2830-2842, 1979

Murphy, J. R., R. M. Haberle, O. B. Toon, and J. B. Pollack, Martian global dust storms: zonally symmetric numerical simulation including size-dependent particle transport, J. Geophys. Res., 98, 3197-3220, 1993.

Nakajima, K., Direct numerical experiments on the large-scale organizations of cumulus convection, Ph.D. Thesis, University of Tokyo, 1994 (in Japanese).

Nakajima, K. and T. Matsuno, Numerical experiments concerning the origin of cloud clusters in the tropical atmosphere, J. Met. Soc. Japan, 66, 309-329, 1988

Ogura, Y. and N. A. Phillips, Scale analysis of deep and shallow convection in the atmosphere, J. Atmos. Sci., 19, 173-179, 1962.

Pollack, J. B., D. S. Colburn, F. M. Flaser, R. Kahn, C. E. Carlston, and D. Pidek, Properties and effects of dust particles suspended in the Martian atmosphere, J. Geophys. Res., 84, 2929-2945, 1979.

Tao, W.-K., S. Lang, J. Simpson, C.-H. Sui, B. Ferrier, and M.-D. Chou, Mechanisms of cloud-radiation interaction in the tropics and midlatitude, J. Atmos. Sci., 53, 2624-2651, 1996.

Vallis, G. K., G. J. Shutts, and M. E. B. Gray, Balanced mesoscale motion and stratified turbulence forced by convection, Q. J. R. Met. Soc., 123, 1621-1652, 1997.

Wilson, R. J. and K. Hamilton, Comprehensive model simulation of thermal tides in the Martian atmosphere, J. Atmos. Sci., 53, 1290-1326, 1996.

Zurek, R. W., J. R. Barnes, R. M. Haberle, J. B. Pollack, J. E. Tillman, and C. B. Leovy, Dynamics of the atmosphere of Mars, in Mars, edited by H. H. Kieffer, B. M. Jakosky, C. W. Snyder, and M. S. Matthews, pp. 835-933, University of Arizona Press, U.S.A., 1992.

M. Odaka (e-mail: odakker@ms.u-tokyo.ac.jp), K. Nakajima (e-mail: ken-suke@deepconv.geo.kyushu-u.ac.jp), S. Takehiro (e-mail: takepiro@geo.kyushu-u.ac.jp), M. Ishiwatari (e-mail: momoko@ ees.hokudai.ac.jp), and Y.Hayashi (e-mail: shosuke@ms.u-tokyo.ac.jp) 